'Instituto Médico Schilkrut, Santiago de Chile.

2Departamento de Psiquiatría y Salud Mental Oriente, Universidad de Chile.

${ }^{3}$ Becado Psiquiatría Adultos, Facultad de Medicina, Universidad de Chile.

${ }^{4}$ Becada Psiquiatría Adultos, Universidad de los Andes.

Santiago, Chile.

Apoyo financiero: No se recibió apoyo financiero para esta investigación.

Recibido el 3 de julio de 2014, aceptado el 10 de noviembre de 2014.

Correspondencia a: Yamil Quevedo Av. Las Condes 10373 of 3,4 y 5 . Las Condes, Santiago.

Teléfonos: 22453850 yamilquevedo@u.uchile.cl

\section{Cambio en el patrón de consumo de sustancias posterior a cirugía bariátrica: presentación de un caso clínico}

\author{
YAMIL QUEVEDO ${ }^{1,2}$, KURT KIRSTEN ${ }^{3}$, \\ CONSUELO PONCE DE LEÓN ${ }^{4}$, ALEJANDRO FERNÁNDEZ ${ }^{1}$
}

\section{Changes in the pattern of substance use after bariatric surgery: report of one case}

Even though the benefits of bariatric surgery are supported by scientific evidence, its indications and contraindications must be revised to avoid its indiscriminate use. Substance use is more common in patients subjected to bariatric surgery than in the general population. After surgery, an increase in alcohol abuse has been reported. We report a 41 years old male, with morbid obesity, alcohol and cocaine use. After bariatric surgery, his alcohol tolerance significantly decreased, increasing the doses of cocaine and starting to consume it without alcohol. His high anxiety level and paranoid delusions, motivated him to seek help in a rehabilitation center where a Substance Dependence Disorder was diagnosed and received initial treatment. The cause of this adverse effect needs further research. Functional and anatomic changes in the digestive tract lead to a greater alcohol absorption and reduced alcohol dehydrogenase activity. Also neurochemical alterations may produce a displacement from compulsive use of food to compulsive use of addictive substances.

(Rev Med Chile 2015; 143: 116-119)

Key words: Alcoholism; Bariatric surgery; Substance-related disorders.
$\mathrm{D}$ istintas publicaciones han reportado un incremento en el consumo de alcohol en pacientes después de cirugía bariátrica ${ }^{11,12}$. Se presenta el caso clínico de un paciente sometido a cirugía bariátrica, con consumo postquirúrgico de alcohol y cocaína y que ingresa a rehabilitación en el Instituto Médico Schilkrut. Se adjunta una revisión de la literatura sobre el tema.

\section{Caso Clínico}

Paciente de sexo masculino, de 41 años de edad, de actividad comerciante, sin antecedentes mórbidos conocidos ni prescripción farmacológica. Presenta un índice de masa corporal (IMC) de 43,5 (159,5 kilos de peso; 1,91 metros de altura), con una ingesta diaria de aproximadamente 4.500 calorías. Paralelamente desde los 25 años de edad, inició consumo ocasional de cocaína aspirada en frecuencia aproximada de $1 \mathrm{~g} / \mathrm{semana}$, en contexto de eventos sociales nocturnos.

El año 2010, fue sometido a evaluación para cirugía bariátrica, en la cual omitió el antecedente de consumo de drogas. El procedimiento quirúrgico realizado fue una manga gástrica sin complicaciones postoperatorias, alcanzando un IMC de 26 (95 kilos de peso). No asistió a apoyo psicológico postoperatorio indicado.

Cinco meses posteriores a la intervención quirúrgica, el paciente refiere una menor tolerancia al alcohol, intoxicándose y alcanzando estado de ebriedad con menor cantidad, lo que se acompaña de un aumento paulatino del consumo de cocaína con la intención de compensar el efecto depresor del alcohol. Previo a la cirugía sólo consumía 
cocaína en instancias sociales; no obstante llega a consumir un total de $10 \mathrm{~g}$ de cocaína por semana y hasta 48 unidades semanales de bebida estándar (U.B.E) en forma solitaria. La elevación del consumo es explicada por el paciente como "al no poder comer, consumía; cambié los alimentos por la cocaína". Este cambio en el patrón de consumo se correlaciona con la aparición de ansiedad significativa, en particular asociada a consecuencias de la conducta adictiva como conflictos de pareja y la pérdida de su trabajo, y además a la manifestación de ideación paranoide en contexto de consumo. Estos síntomas motivaron en 2012, la consulta al equipo de salud mental y se decidió su hospitalización psiquiátrica. Se diagnosticó un Trastorno por Dependencia a Alcohol y Cocaína y se inició el proceso de desintoxicación y rehabilitación, cursando con evolución favorable, completando a la fecha 10 meses de tratamiento, manteniendo abstinencia confirmada con exámenes de orina semanales, estabilidad de su ánimo, mejora en las relaciones interpersonales y motivación para la mantención de abstinencia y estilo de vida saludable a largo plazo.

\section{Discusión}

La elevada prevalencia de obesidad en el mundo y su asociación con enfermedades metabólicas han incentivado la búsqueda de alternativas terapéuticas que permitan alcanzar resultados duraderos y efectivos a dicha condición de carácter epidémico. De esta manera, durante las últimas décadas, se han propuesto una variedad de procedimientos quirúrgicos destinados a la reducción del peso ${ }^{1,2}$. Si bien sus beneficios han sido respaldados por una serie de estudios ${ }^{3}$, la consideración de indicaciones precisas y contraindicaciones resultan fundamentales para evitar un uso indiscriminado y potencialmente perjudicial.

Se observa en el caso presentado, una omisión voluntaria por parte del paciente del antecedente de uso habitual de alcohol y drogas durante su evaluación preoperatoria, conducta esperable en personas con Trastorno por Dependencia ya que estos tienden a negar o minimizar su consumo ${ }^{4}$, determinando por tanto, que este dato no sea conocido por el equipo clínico que evalúa la indicación de la cirugía. Al respecto, el abuso o dependencia activa de sustancias ha sido conside- rado como una de tan solo cuatro contraindicaciones claras para cirugía bariátrica de acuerdo a guías y consensos internacionales, recomendando además una evaluación psicológica rutinaria a los candidatos ${ }^{5}$. Aunque numerosos reportes han mencionado la alta probabilidad de psicopatología en pacientes en espera de resolución quirúrgica, los resultados tienden a ser dispares y son pocos los que han utilizado entrevistas estructuradas ${ }^{6}$. Trabajos recientes han sugerido que tener una historia previa de trastornos relacionados al consumo de sustancias es más frecuente en aquellos que consultan por cirugía bariátrica en comparación al resto de la población ${ }^{7,8}$. Basado en entrevistas clínicas estructuradas, se ha identificado una prevalencia de vida para patología del Eje I del $50,5 \%$, ubicando al abuso de alcohol como el tercer trastorno más común en candidatos a cirugía para reducción de peso, por debajo del trastorno depresivo y trastorno de pánico ${ }^{8,9}$.

En el caso clínico expuesto, posterior a la operación, se consiguió en 5 meses de evolución, un cambio significativo en el patrón de consumo, destacando cuatro hechos clínicamente relevantes: la disminución de la tolerancia al alcohol, el incremento en las dosis y frecuencia del uso de cocaína, la transición desde el consumo en instancias sociales al consumo en solitario y finalmente la aparición de sintomatología ansiosa y paranoidea. En relación a estos cambios, existen reportes de aumento en la incidencia del uso inadecuado y abuso de alcohol en aquellos pacientes sometidos a cirugía bariátrica ${ }^{10}$. Se ha estimado que entre un 2 a $6 \%$ de los ingresos a centros de tratamiento para adicciones tendrían historia de cirugía bariátrica anterior $^{11}$. Estudios de seguimiento a largo plazo han encontrado un aumento en las tasas de abuso de alcohol en pacientes post-operados $(2,6 \%$ antes versus $5,1 \%$ después de cirugía $)^{12}$. Otro estudio, de tipo cohorte prospectivo, evidencia que al segundo año de seguimiento post cirugía, la prevalencia de trastornos asociados al uso de alcohol resulta significativamente mayor $(9,6 \%)$ respecto al año anterior o al momento previo a la intervención. En este mismo estudio, se identifican como probables factores de riesgo, el sexo masculino, menor edad, historia previa de problemas con el uso de alcohol y haber sido sometido a bypass gástrico en Y de Roux (BGYR), entre otros ${ }^{13}$. Producto de las diferencias en el riesgo de uso de alcohol que representan las distintas técnicas quirúrgicas, se 
ha sugerido que los cambios anátomo-funcionales podrían verse relacionados con las alteraciones farmacocinéticas que llevan a la intoxicación. Se ha reportado un aumento en la velocidad de absorción, disminución del intervalo de tiempo para alcanzar concentraciones máximas, aumento de las concentraciones máximas y un incremento de los tiempos de eliminación de alcohol ${ }^{10}$. Estudios realizados en Chile, en pacientes sometidos a gastrectomía vertical ("manga gástrica") han sido coincidentes en demostrar que los cambios anatómicos digestivos modifican considerablemente la absorción de alcohol alcanzando niveles plasmáticos más altos y persistentes ${ }^{14}$. Se ha relacionado el rápido vaciamiento gástrico al aumento en la velocidad de absorción y la reducción del volumen del estómago a menor cantidad de alcohol deshidrogenasa, afectando el metabolismo de la sustancia ${ }^{15}$. Por su parte, la marcada disminución del IMC podría ser un factor a considerar si las cantidades de consumo previo se mantienen ${ }^{16}$. Sin embargo, no ha sido encontrada una asociación consistente entre la baja de peso y el desarrollo de trastorno por uso de alcohol postquirúrgico ${ }^{17}$. La malabsorción de vitamina B12 en sujetos operados y además consumidores de alcohol supone un riesgo agregado de complicaciones metabólicas que debe ser considerado.

Por otro lado, grupos de investigación se han interesado en cómo pacientes obesos y sometidos a cirugía de reducción de peso, han remplazado sus conductas de comer en exceso por otras adicciones como el uso de alcohol u otras drogas; conductas de juego patológico u otras como ejercicio excesivo y compras compulsivas luego de la cirugía. Basados en modelos animales y la evidencia existente de estudios en humanos, se han evaluado similitudes neuroquímicas que relacionan formas compulsivas de alimentación con otras conductas compulsivas, sugiriendo que la primera en pacientes obesos, actúa como factor protector de otras conductas como recompensa de drogas y otras conductas adictivas. De esta forma se ha sugerido que la privación de azúcar provoca desbalances en neurotransmisores similares a estados de privación de opioides ${ }^{18}$. Así, se ha acuñado el término de "síndrome de deficiencia de la recompensa" (SDR) referido a la insuficiencia de sentimientos habituales de satisfacción y que serían resultado de una disfunción de ciertas cascadas cerebrales relacionadas a la recompensa que involucran interacción entre diferentes neurotransmisores, principalmente dopamina y opioides ${ }^{19}$. Dichos elementos estarían a la base del cambio de la "adicción a la comida" por otras "adicciones" (fenómeno conocido como "transferencia de la adicción") que permiten explicar, desde otra perspectiva, el aumento del consumo de sustancias de pacientes obesos sometidos a cirugía bariátrica ${ }^{18} y$ que en el caso clínico expuesto corresponderían a alcohol y cocaína.

Finalmente, consideramos necesario fortalecer las evaluaciones e intervenciones preoperatorias y de seguimiento que existen actualmente y que permitan disminuir los riesgos relacionados al consumo de sustancias en este grupo de pacientes. Se han sugerido recomendaciones con énfasis en el tamizaje inicial, reconocimiento de subgrupos de mayor riesgo, consideración de la historia familiar, consentimiento informado y sesiones psicoeducativas respecto al riesgo del uso de sustancias postcirugía ${ }^{10}$. Esta última intervención siendo especialmente reconocida como necesaria por los propios pacientes ${ }^{20}$.

Dado lo anterior, nos parece razonable considerar, debido a la complejidad de alguna de estas intervenciones, la participación de profesionales especialistas en adicciones en pacientes de mayor riesgo.

\section{Referencias}

1. NIH conference. Gastrointestinal surgery for severe obesity. Consensus Development Conference Panel. Ann Intern Med 1991;115 (12): 956-61.

2. Smoot TM, Xu P, Hilsenrath P, Kuppersmith NC, Singh KP. Gastric bypass surgery in the United States, 19982002. Am J Pub Health 2006; 96 (7): 1187-9.

3. Colquitt JL, Picot J, Loveman E, Clegg AJ. Surgery for obesity. Cochrane Database Syst Rev 2009; 15 (2): CD003641.

4. Goldstein RZ, Craig AD, Bechara A, Garavan H, Childress A, Paulus MP, et al. The Neurocircuitry of Impaired Insight in Drug Addiction. Trends Cogn Sci 2009; 13 (9): 372-80.

5. Mechanick JI, Kushner RF, Sugerman HJ, GonzálezCampoy JM, Collazo-Clavell ML, Spitz AF, et al. American Association of Clinical Endocrinologists, the Obesity Society, and American Society for Metabolic \& Bariatric Surgery medical guidelines for clinical practice for the perioperative nutritional, metabolic, and nonsurgical support of the bariatric surgery patient. Obesity (Silver 
Spring) 2009; 17 (Suppl 1): S1-70.

6. Mitchell JE, Selzer F, Kalarchian MA, Devlin MJ, Strain GW, Elder KA, et al. Psychopathology before surgery in the Longitudinal Assessment of Bariatric Surgery-3 (LABS-3) psychosocial study. Surg Obes Relat Dis 2012; 8 (5): 533-41.

7. Kalarchian MA, Marcus MD, Levine MD, Courcoulas AP, Pilkonis PA, Ringham RM, et al. Psychiatric disorders bariatric surgery candidates: relationship to obesity and functional health status. Am J Psychiatry 2007; 164 (2): 328-34.

8. Substance Abuse and Mental Health Services Administration. Results from the 2008 national survey on drug use and health: national findings. Rockville, MD: Office of Applied Studies, NSDUH Series H-36, HHS Publication No. SMA 09-4434.

9. Jones-Corneille LR, Wadden TA, Sarwer DB, Faulconbridge LF, Fabricatore AN, Stack RM, et al. Axis I psycho- pathology in bariatric surgery candidates with and without binge eating disorder: results of structured clinical interviews. Obes Surg 2012; 22 (3): 389-97.

10. Ashton K, Heinberg L, Merrell J, Lavery M, Windover A, Alcorn K. Pilot evaluation of a substance abuse prevention group intervention for at-risk bariatric surgery candidates. Surg Obes Relat Dis 2013; 9 (3): 462-7.

11. Saules KK, Wiedemann A, Ivezaj V, Hopper JA, FosterHartsfield J, Schwarz D. Bariatric surgery history among substance abuse treatment patients: prevalence and associated features. Surg Obes Relat Dis 2010; 6 (6): 615-21.

12. Mitchell JE, Lancaster KL, Burgard MA, Howell LM, Krahn DD, Crosby RD, et al. Long-term follow-up of patients' status after gastric bypass. Obes Surg 2001; 11
(4): 464-8.

13. King WC, Chen JY, Mitchell JE, Kalarchian MA, Steffen KJ, Engel SG, et al. Prevalence of alcohol use disorders before and after bariatric surgery. JAMA 2012; 307(23): 2516-25.

14. Maluenda F, Csendes A, De Aretxabala X, Poniachik J, Salvo K, Delgado I, et al. Alcohol absorption modification after a laparoscopic sleeve gastrectomy due to obesity. Obes Surg 2010; 20 (6): 744-8.

15. Hagedorn JC, Encarnación B, Brat GA, Morton JM. Does gastric bypass alter alcohol metabolism? Surg Obes Relat Dis 2007; 3 (5): 543-8.

16. Klockhoff H, Näslund I, Jones AW. Faster Absorption of Ethanol and Higher Peak Concentration in Women after Gastric Bypass Surgery, Br J Clin Pharmacol 2002; 54 (6): 587-91.

17. Suzuki J, Haimovici F, Chang G. Alcohol use disorders after bariatric surgery, Obes Surg 2012; 22 (2): 201-7.

18. Blum K, Bailey J, González AM, Oscar-Berman M, Liu Y, Giordano J, et al. Neuro-genetics of Reward Deficiency Syndrome (RDS) as the root cause of "addiction Transfer": A new phenomenon common after bariatric surgery. J Genet Syndr Gene Ther 2011; (1): S2-001.

19. Blum K, Gardner E, Oscar-Berman M, Gold M. "Liking” and "wanting" linked to Reward Deficiency Syndrome (RDS): hypothesizing differential responsivity in brain reward circuitry. Curr Pharm Des 2012; 18 (1): 113-8.

20. Ivezaj V, Saules K, Wiedemann A. “I Didn't See This Coming: Why Are Postbariatric Patients in Substance Abuse Treatment? Patients' Perceptions of Etiology and Future Recommendations. Obes Surg 2012; 22 (8): 1308-14. 